\title{
Inertial-Alfvén Waves as Columnar Helices in Planetary Cores
}

\author{
O. P. Bardsley ${ }^{1} \dagger$ and P. A. Davidson ${ }^{1}$ \\ ${ }^{1}$ Department of Engineering, University of Cambridge, \\ Trumpington Street, Cambridge, CB2 1PZ, UK
}

(Received $\mathrm{xx}$; revised $\mathrm{xx}$; accepted $\mathrm{xx}$ )

We consider a rapidly-rotating, Boussinesq fluid stirred by buoyant anomalies. In such a system it is known that, in the absence of a magnetic field, inertial waves whose wavevectors lie normal to the rotation axis play a key role in establishing quasi-geostrophic motion. In particular, buoyant anomalies radiate low-frequency inertial wave packets which disperse along the rotation axis, leading to axially elongated columnar vortices. Here we focus on the influence of an ambient magnetic field on this process, motivated by the dynamics of planetary cores. We find that, once again, the waves responsible for establishing quasi-geostrophic structures have wave-vectors normal to the rotation axis; however, these are not conventional inertial waves, but rather hybrid "inertial-Alfvén waves". Their frequency equals that of an Alfvén wave but their axial group velocity is half that of the equivalent inertial wave. They have maximal kinetic, magnetic and cross helicity, carry magnetic and kinetic energy in equal amounts, and are particularly potent in establishing columnar, helical vortices through the spontaneous emission of axially elongated wave packets. Although our hybrid inertial-Alfvén waves have been overlooked in dynamo literature to date, we speculate that they in fact play a central role in planetary dynamos.

\section{Key words:}

\section{Introduction}

1.1. Columnar vortex formation in the absence of a magnetic field

One of the most striking features of recent numerical simulations of planetary dynamos is the ubiquitous appearance of sinuous columnar vortices aligned with the rotation axis and packed with intense kinetic helicity, usually negative in the north and positive in the south. Such helical vortices are essential to maintaining dynamo action in the numerical dynamos (Christensen 2011). The manner in which these columnar flows spontaneously emerge from buoyant anomalies is now reasonably well understood, at least in the absence of a significant Lorentz force. So, before considering the influence of a magnetic field, perhaps it is useful to review the non-magnetic case.

Consider a rapidly-rotating Boussinesq fluid stirred by buoyant anomalies which slowly drift under the influence of gravity. To focus thoughts we take the background rotation, $\boldsymbol{\Omega}=\boldsymbol{\Omega} \boldsymbol{e}_{z}$, and gravitational acceleration, $\boldsymbol{g}$, to be mutually orthogonal and adopt a coordinate system rotating with the fluid; the structure of the helical columnar vortices generated by localised buoyant blobs in such an environment is discussed in detail in

$\dagger$ Email address for correspondence: ob275@eng.cam.ac.uk 
Davidson (2014). Since the blob constitutes a disturbance in a rapidly-rotating system, it is obliged to spontaneously radiate a spectrum of inertial waves with angular frequency $\varpi= \pm 2 \boldsymbol{k} \cdot \boldsymbol{\Omega} / k$ and group velocity $\boldsymbol{c}_{g}= \pm 2(\boldsymbol{k} \times(\boldsymbol{\Omega} \times \boldsymbol{k})) / k^{3}$. Here the upper (lower) sign corresponds to upward (downward) propagating waves with negative (positive) helicity, $\boldsymbol{k}$ is the wave-vector and $k=|\boldsymbol{k}| \sim \pi / \ell$ for some blob scale $\ell$. It transpires that the resulting dispersion pattern is dominated by a pair of columnar vortices above the blob, one cyclonic and one anti-cyclonic, matched to a cyclone-anticyclone pair beneath it. All four columnar vortices elongate along the rotation axis at a rate governed by the group velocity of low-frequency $\left(\boldsymbol{k} \cdot \boldsymbol{\Omega} \approx 0\right.$ ) inertial waves, $\boldsymbol{c}_{g}= \pm 2 \Omega / k$, with the upwardpropagating waves carrying negative helicity and the downward ones positive helicity. This antisymmetric helicity distribution is consistent with the results of numerical simulations, in which buoyant anomalies outside of the tangent cylinder have a tendency to concentrate near to the equator, and the associated helicity is negative in the north and positive in the south (Davidson \& Ranjan 2015).

The reason why the radiation pattern is dominated by wave packets propagating along the rotation axis is discussed in Davidson et al. (2006). A disturbance of arbitrary shape will, of course, excite waves with a broad spectrum of wave-vectors, and the direction of propagation of the resulting waves will vary, being fixed by the orientation of $\boldsymbol{k}$ through the expression for $\boldsymbol{c}_{g}$. However, the fact that $\boldsymbol{c}_{g}$ is always perpendicular to $\boldsymbol{k}$ implies a special role for the low-frequency $(\boldsymbol{k} \cdot \boldsymbol{\Omega} \approx 0)$ waves in this dispersion, as all the energy associated with these wave-vectors (which lie in the transverse plane) is radiated along the rotation axis. In short, all the energy contained in a thin horizontal disc in $\boldsymbol{k}$-space propagates along a narrow cylinder in real space, and the process of channelling energy from a two-dimensional object (a thin disc) to a one-dimensional object (a narrow cylinder) amplifies the radiation density in axially-travelling wave packets (Davidson 2013). Put another way, if we consider an imaginary cylinder aligned with $\Omega$ and circumscribing the buoyant blob, there is one and only one orientation of $\boldsymbol{k}$ which transports energy from the blob to a point outside of the cylinder, but an infinity of wave-vectors which carry energy from the blob to a given point within it.

\subsection{The addition of a magnetic field and the magnetostrophic wave regime}

In planetary dynamos the magnetic field is dynamically active and so there is a long history of investigating how a magnetic field modifies inertial waves (e.g. Hide 1966; Finlay et al. 2010). We now summarise this - in part to establish notation and in part to distinguish our findings from previous work. Suppose that, in the rotating reference frame, there is a steady, uniform magnetic field $\boldsymbol{B}_{0}$, which we take to be orthogonal to $\boldsymbol{\Omega}$. (In the case of the earth, this could represent the local east-west field.) If we consider small perturbations to a quiescent state, the linearised equations of motion for an ideal fluid are (ignoring for a moment the buoyant source term):

$$
\frac{\partial \boldsymbol{b}}{\partial t}=\left(\boldsymbol{B}_{0} \cdot \nabla\right) \boldsymbol{u}, \quad \frac{\partial \boldsymbol{u}}{\partial t}=\left(\boldsymbol{B}_{0} \cdot \nabla\right) \boldsymbol{b}+(2 \boldsymbol{\Omega} \cdot \nabla) \boldsymbol{c}, \quad \nabla \times \boldsymbol{a}=\boldsymbol{b}, \quad(1.1 a, b, c)
$$

where $\boldsymbol{u}$ and $\boldsymbol{b}$ are the velocity and magnetic perturbation fields, $\boldsymbol{c}$ and $\boldsymbol{a}$ are their respective vector potentials, and all four fields are solenoidal. We scale the magnetic field to have dimensions of an Alfvén velocity by taking the density and permeability to be unity. The governing equations combine to give the wave-like equation

$$
\left[\frac{\partial^{2}}{\partial t^{2}}-\left(\boldsymbol{B}_{0} \cdot \nabla\right)^{2}\right]^{2} \nabla^{2} \boldsymbol{u}+(2 \boldsymbol{\Omega} \cdot \nabla)^{2} \frac{\partial^{2} \boldsymbol{u}}{\partial t^{2}}=\mathbf{0} .
$$


For $\boldsymbol{B}_{0}=\mathbf{0}$ we recover inertial waves, whilst $\boldsymbol{\Omega}=\mathbf{0}$ gives Alfvén waves with a frequency $\varpi=( \pm) \boldsymbol{k} \cdot \boldsymbol{B}_{0}$ and group velocity $\boldsymbol{c}_{g}=( \pm) \boldsymbol{B}_{0}$. Searching for plane-wave solutions to (1.2) in the form $\boldsymbol{u}=\hat{\boldsymbol{u}} \exp \{\mathrm{i}(\boldsymbol{k} \cdot \boldsymbol{x}-\varpi t)\}$ yields

$$
\hat{\boldsymbol{\omega}}=\mp k \hat{\boldsymbol{u}}, \quad \hat{\boldsymbol{j}}=\mp k \hat{\boldsymbol{b}}, \quad \hat{\boldsymbol{b}}=-\frac{\varpi_{B}}{\varpi} \hat{\boldsymbol{u}},
$$

and the dispersion relationship

$$
\varpi^{2} \mp \varpi_{\Omega} \varpi-\varpi_{B}^{2}=0,
$$

where $\varpi_{B}=\boldsymbol{k} \cdot \boldsymbol{B}_{0}, \varpi_{\Omega}=2 \boldsymbol{k} \cdot \boldsymbol{\Omega} / k, \boldsymbol{\omega}$ is the vorticity field and $\boldsymbol{j}$ is the current density. Evidently, $\boldsymbol{u}$ and $\boldsymbol{\omega}$ are in phase and aligned, as are $\boldsymbol{b}$ and $\boldsymbol{j}$, implying all plane-wave solutions of (1.2) have maximal kinetic and magnetic helicity $(\boldsymbol{u} \cdot \boldsymbol{\omega}$ and $\boldsymbol{a} \cdot \boldsymbol{b})$, with the upper sign in (1.3) corresponding to negative helicity. Moreover, the fact that $\hat{\boldsymbol{\omega}}=\mp k \hat{\boldsymbol{u}}$, irrespective of the presence of a mean magnetic field, tells us that all monochromatic solutions of (1.2) have the same spatial structure for $\boldsymbol{u}$, which turns out to be a circularly polarised transverse wave (Moffatt 1978).

The group velocity corresponding to (1.4) can be expressed as

$$
\left[1+\left(\frac{\varpi_{B}}{\varpi}\right)^{2}\right] \boldsymbol{c}_{g}=\frac{2 \varpi_{B}}{\varpi} \boldsymbol{B}_{0} \pm \boldsymbol{c}_{g \Omega},
$$

where $\boldsymbol{c}_{g \Omega}=2(\boldsymbol{k} \times(\boldsymbol{\Omega} \times \boldsymbol{k})) / k^{3}$. Note that, as for inertial waves, all wave vectors in the horizontal plane dispatch energy along the rotation axis. Note also that

$$
\left[1+\left(\frac{\varpi_{B}}{\varpi}\right)^{2}\right] \boldsymbol{c}_{g} \cdot \boldsymbol{\Omega}= \pm \boldsymbol{c}_{g \Omega} \cdot \boldsymbol{\Omega}= \pm 2 k^{-3}\left[(k \Omega)^{2}-(\boldsymbol{k} \cdot \boldsymbol{\Omega})^{2}\right],
$$

so wave packets propagating in the direction of $\boldsymbol{\Omega}$ carry negative kinetic and magnetic helicity, whilst those propagating antiparallel to $\Omega$ carry positive helicity.

The geodynamo is expected to operate in the rapidly-rotating regime $\Omega l \gtrsim 10|\boldsymbol{B}|$ so that, if we ignore the degenerate case of $\boldsymbol{k} \cdot \boldsymbol{\Omega} \approx 0$, we have $\left|\varpi_{\Omega}\right| \gg\left|\varpi_{B}\right|$ and the dispersion relationship (1.4) has two well-separated roots: $\varpi \approx \pm \varpi_{\Omega}$ and $\varpi \approx$ $\mp \varpi_{B}^{2} / \varpi_{\Omega}$. The first represents fast weakly modified inertial waves and the second gives slow magnetostrophic waves, which have been much discussed in the context of planetary cores (e.g. Braginsky 1967; Moffatt 1978) owing to their low frequency and slow group velocity. However, with the discussion of section 1.1 in mind, one might expect the special case of $\boldsymbol{k} \cdot \boldsymbol{\Omega} \approx 0$ to play a central role in the formation of columnar structures - yet these waves do not belong to either the inertial or magnetostrophic regimes.

\section{Hybrid inertial-Alfvén waves}

Consider now those waves which satisfy $\boldsymbol{k} \cdot \boldsymbol{\Omega} \approx 0$, so that $\left|\varpi_{\Omega}\right| \ll\left|\varpi_{B}\right|$ despite the fact that $\Omega l \gtrsim 10|\boldsymbol{B}|$. To leading order in $\left|\varpi_{\Omega}\right| /\left|\varpi_{B}\right|,(1.4)$ and (1.5) yield

$$
\varpi=( \pm) \varpi_{B} \pm \frac{\varpi_{\Omega}}{2} \approx( \pm) \varpi_{B}, \quad c_{g}=( \pm) \boldsymbol{B}_{0} \pm \frac{\Omega}{k},
$$

where the upper or lower sign in \pm may be chosen independently to that in $( \pm)$. These are hybrid waves with the frequency of Alfvén waves, which propagate accordingly along field lines, but also disperse energy along the rotation axis at half the speed of low-frequency inertial waves. As (1.3) still holds, they have maximal kinetic, magnetic and cross helicity, and equipartition of energy, $\hat{\boldsymbol{u}}=(\mp) \hat{\boldsymbol{b}}$. In the earth's core the axial group velocity of these waves is extremely fast; for example, taking $l \sim 10 \mathrm{~km}$, hybrid inertial-Alfvén waves can 
traverse the core in months. By way of contrast, pure Alfvén waves would traverse the core in decades, whereas convective motions and magnetostrophic waves have time scales measured in centuries.

Since $\boldsymbol{k} \cdot \boldsymbol{\Omega} \approx 0$, rotation appears at leading order in the expression for $\boldsymbol{c}_{g}$ but not in that for $\varpi$. From a strictly kinematic point of view, one may conceive of these hybrid waves as a pair of linearly polarised Alfvén waves propagating horizontally along $\boldsymbol{B}_{0}$ and superimposed at right angles with a $\pi / 2$ phase shift to yield the circularly polarised wave demanded by (1.3). From (2.1) we see that, like low-frequency inertial waves, they focus radiated energy onto the rotation axis and, when the waves emanate from a localised source of buoyancy, this focussing of energy will produce a particularly high energy density within the axially-propagating wave packets. We expect, therefore, that these hybrid waves will assume the role adopted by low-frequency inertial waves in the strictly hydrodynamic case, with localised buoyant blobs spontaneously radiating axiallyelongated wave packets which eventually evolve into quasi-geostrophic vortices.

The curious fact that the axial group velocity is halved in comparison with the equivalent low-frequency inertial waves can be understood as follows. From (1.1) we have the general energy-density energy-flux relationship

$$
\frac{\partial e}{\partial t}=\nabla \cdot\left[(\boldsymbol{u} \cdot \boldsymbol{b}) \boldsymbol{B}_{0}+(\boldsymbol{u} \cdot \boldsymbol{c}) \boldsymbol{\Omega}+\boldsymbol{c} \times(\boldsymbol{\Omega} \cdot \nabla \boldsymbol{c})\right],
$$

where $e=\frac{1}{2}\left(\boldsymbol{u}^{2}+\boldsymbol{b}^{2}\right)$ is the energy density. For axially elongated structures we expect the final term in the divergence to vanish, so the first two terms represent transverse and axial contributions to the energy flux, with the axial energy flux being $-(\boldsymbol{u} \cdot \boldsymbol{c}) \boldsymbol{\Omega}$. For the case of a monochromatic wave, (1.3) requires $\hat{\boldsymbol{u}}=\mp k \hat{\boldsymbol{c}}$, from which $\nabla \cdot[\boldsymbol{c} \times(\boldsymbol{\Omega} \cdot \nabla \boldsymbol{c})]=0$ and the axial energy flux becomes $\pm \boldsymbol{u}^{2}(\boldsymbol{\Omega} / k)$. In the case of low-frequency inertial waves, where $e=\boldsymbol{u}^{2} / 2$, this yields the expected axial group velocity of $\pm 2 \boldsymbol{\Omega} / k$, whereas for our hybrid inertial-Alfvén waves (for which $e=\boldsymbol{u}^{2}$ ) the resulting axial group velocity is $\pm \boldsymbol{\Omega} / k$, as in $(2.1 \mathrm{~b})$.

Alternatively we may rewrite (1.1) in terms of Elsasser variables $\boldsymbol{v}^{(\mp)}=\boldsymbol{u}(\mp) \boldsymbol{b}$ and their corresponding vector potentials $\kappa^{(\mp)}$, to give

$$
\frac{\partial \boldsymbol{v}^{(\mp)}}{\partial t}=(\mp)\left(\boldsymbol{B}_{0} \cdot \nabla\right) \boldsymbol{v}^{(\mp)}+(\boldsymbol{\Omega} \cdot \nabla)\left(\boldsymbol{\kappa}^{+}+\boldsymbol{\kappa}^{-}\right) \text {. }
$$

for a hybrid wave travelling parallel to $\boldsymbol{B}_{0}$ we have $\boldsymbol{v}^{+}=\mathbf{0}$, whilst one travelling antiparallel has $\boldsymbol{v}^{-}=\mathbf{0}$; in either case, our hybrid waves are governed by

$$
\frac{\partial \boldsymbol{v}^{(\mp)}}{\partial t}=(\mp)\left(\boldsymbol{B}_{0} \cdot \nabla\right) \boldsymbol{v}^{(\mp)}+(\boldsymbol{\Omega} \cdot \nabla) \boldsymbol{\kappa}^{(\mp)} \text {. }
$$

Note the absence of the pre-multiplying factor of two seen in (1.1b). The dispersion relationship for (2.4) is readily shown to be $\varpi=( \pm) \varpi_{B} \pm \frac{1}{2} \varpi_{\Omega}$, as in (2.1a).

\section{The spontaneous radiation of waves from a localised source}

Consider the somewhat artificial case of waves radiated from a localised buoyant source introduced into the fluid at time $t=0$. We restrict ourselves to the rapidly-rotating regime $\Omega l \gg|\boldsymbol{B}|$ thought to be typical of the earth's core.

From the preceding discussion we expect at least three types of waves to emerge from the localised source. For those waves which satisfy $\left|\varpi_{\Omega}\right| \gg\left|\varpi_{B}\right|$, which will hold for all wave-vectors except those aligned with the transverse plane $(\boldsymbol{k} \cdot \boldsymbol{\Omega} \approx 0)$, we have both fast inertial and slow magnetostrophic waves. Conversely, those which satisfy the 
reverse criterion $\left|\varpi_{\Omega}\right| \ll\left|\varpi_{B}\right|$, which requires $\boldsymbol{k} \cdot \boldsymbol{\Omega} \rightarrow 0$ for $\Omega l \gg|\boldsymbol{B}|$, constitute hybrid inertial-Alfvén waves. (We leave aside the intermediate case $\left|\varpi_{\Omega}\right| \sim\left|\varpi_{B}\right|$, which is not readily classified and in any event unimportant, as we shall see.) Of these three classes of wave, the fastest group velocity belongs to those inertial waves for which $\boldsymbol{c}_{g}$ is closely aligned with the rotation axis, though not so close they violate the criterion $\left|\varpi_{\Omega}\right| \gg\left|\varpi_{B}\right|$. These have an axial group velocity approaching $\boldsymbol{c}_{g} \approx \pm 2 \Omega / k$. Next fastest are the hybrid waves, with roughly half the axial group velocity of the fastest inertial waves, $\boldsymbol{c}_{g}= \pm \boldsymbol{\Omega} / k$. Unlike inertial waves, these transport both magnetic energy and magnetic helicity. Finally, we have the sluggish magnetostrophic waves which emerge slowly and can exhibit a significant energy flux along $\boldsymbol{B}_{0}$.

Of these three wave classes, hybrid inertial-Alfvén waves are the best candidates for establishing quasi-geostrophic structures. This is partly because they are fast, unlike magnetostrophic waves, and partly because they automatically focus energy onto the rotation axis, unlike the off-axis inertial waves which disperse radially. A simple example is sufficient to demonstrate these trends.

\section{An illustrative example}

Consider the case in which $\boldsymbol{\Omega}=\Omega \boldsymbol{e}_{z}, \boldsymbol{B}_{0}=B_{0} \boldsymbol{e}_{y}$ and $\boldsymbol{g}=-g \boldsymbol{e}_{x}$ and the source is a static buoyant density anomaly of Gaussian profile centred on the origin, with a density perturbation $\rho^{\prime} \propto-\exp \left\{-2|\boldsymbol{x}|^{2} / \ell^{2}\right\}$; this has been previously studied in the quasi-static regime (Moffatt \& Loper 1994; St Pierre 1996; Chulliat et al. 2004). The scale $\ell$ is the effective radius of the buoyant blob and the dominant wavenumber for such a disturbance is readily shown to be $k \approx \pi / \ell$. It is convenient to introduce the ratio of Alfvén to inertial frequencies as the dimensionless Lehnert number, $L e=2 B_{0} / \Omega \ell$, which is approximately $2 B_{0} k / \pi \Omega$ and of order 0.1 for small scales in the earth. The vorticity equation is then

$$
\frac{\partial \boldsymbol{\omega}}{\partial t}=\left(\boldsymbol{B}_{0} \cdot \nabla\right) \boldsymbol{j}+(2 \boldsymbol{\Omega} \cdot \nabla) \boldsymbol{u}+\nabla \rho^{\prime} \times \boldsymbol{g}
$$

The wave-like equation for $\boldsymbol{u}$ continues to be (1.2), whilst that for $\boldsymbol{b}$ acquires a source term:

$$
\left[\frac{\partial^{2}}{\partial t^{2}}-\left(\boldsymbol{B}_{0} \cdot \nabla\right)^{2}\right]^{2} \nabla^{2} \boldsymbol{b}+(2 \boldsymbol{\Omega} \cdot \nabla)^{2} \frac{\partial^{2} \boldsymbol{b}}{\partial t^{2}}=\left(\boldsymbol{B}_{0} \cdot \nabla\right)^{3}\left[(\boldsymbol{g} \cdot \nabla) \nabla \rho^{\prime}-\boldsymbol{g} \nabla^{2} \rho^{\prime}\right] .
$$

We solve (1.2) and (4.2) subject to the initial conditions $\boldsymbol{u}=\boldsymbol{b}=\mathbf{0}$, and the associated initial requirements $\partial_{t} \boldsymbol{\omega}=\nabla \rho^{\prime} \times \boldsymbol{g}$ and $\partial_{t}^{2} \nabla^{2} \boldsymbol{u}=(2 \boldsymbol{\Omega} \cdot \nabla)\left(\boldsymbol{g} \times \nabla \rho^{\prime}\right)$, both of which follow from (4.1). The solution procedure is straightforward in principle: we take the spatial Fourier transforms of (1.2) and (4.2) and solve the resulting equations in Fourier space, subject to the initial conditions. This yields a transformed velocity of

$$
\begin{aligned}
& \hat{\boldsymbol{u}}(\boldsymbol{k}, t)=\hat{\rho}^{\prime}\left(\varpi_{\Omega}^{2}+4 \varpi_{B}^{2}\right)^{-\frac{1}{2}}\left[\left(\cos \varpi_{F} t-\cos \varpi_{S} t\right) \boldsymbol{e}_{k} \times \boldsymbol{g}\right. \\
& \left.+\left(\sin \varpi_{F} t+\sin \varpi_{S} t\right) \boldsymbol{e}_{k} \times\left(\boldsymbol{g} \times \boldsymbol{e}_{k}\right)\right],
\end{aligned}
$$

where $\hat{\rho}^{\prime} \propto-\exp \left\{-k^{2} \ell^{2} / 8\right\}, 2 \varpi_{F / S}=\sqrt{\varpi_{\Omega}^{2}+4 \varpi_{B}^{2}} \pm \varpi_{\Omega}$ and $\boldsymbol{e}_{k}=\boldsymbol{k} / k$. The inverse transform, which takes the form of a dispersion integral, is then evaluated numerically.

In the interests of brevity we restrict our discussion to the case $L e=0.1$. Figure 1 shows contours of $u_{z}$, the inverse transform of (4.3), for $z>0$ at $\Omega t=50$, with the righthand panel showing a three-dimensional rendering, the central panel showing the velocity in the plane $x=0$, and the left-hand panel showing the non-magnetic case $(L e=0)$ for comparison. The dispersion pattern in figure $1 \mathrm{~b}$ resembles that which emerges from a 

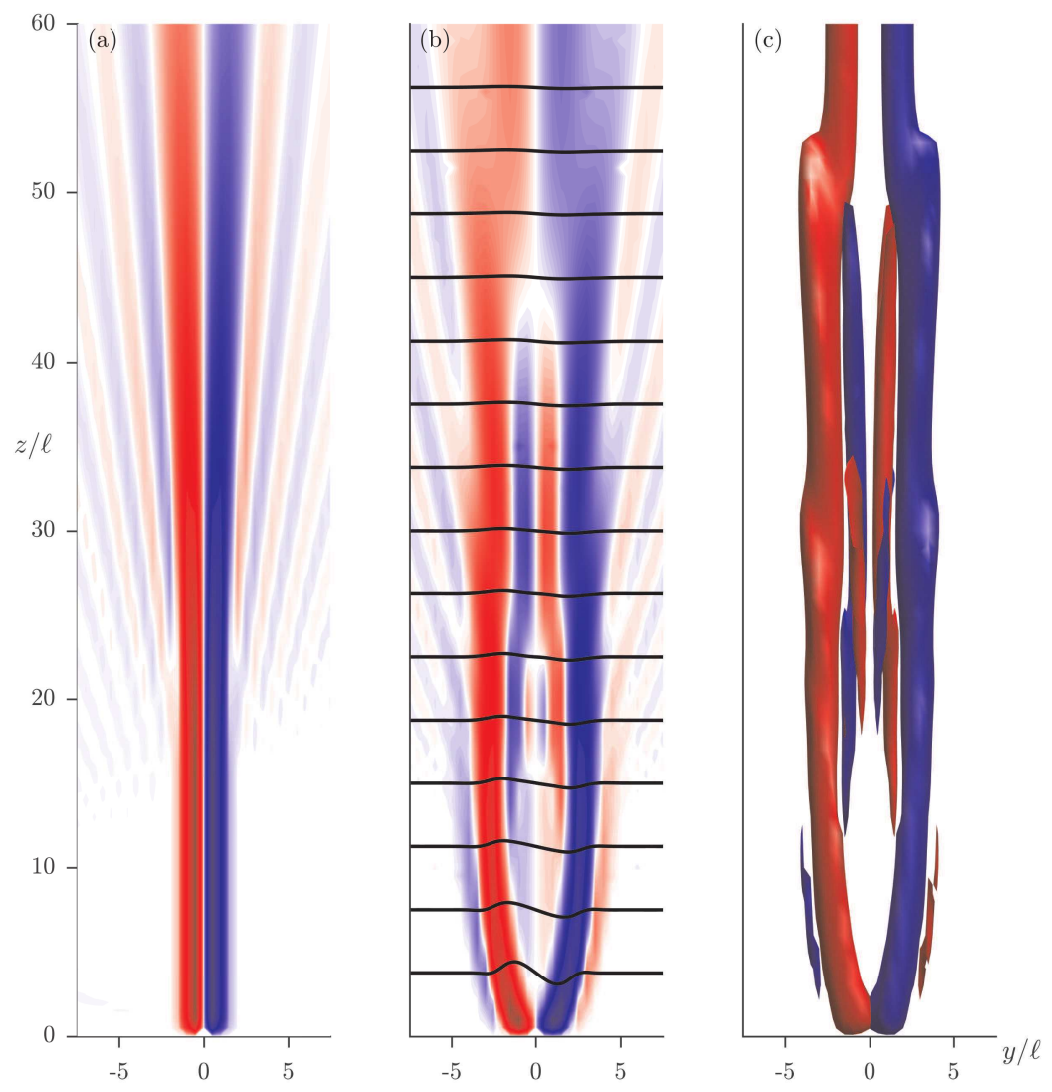

Figure 1. Axial velocity $u_{z}$ at $\Omega t=50$. Rotation is vertical and gravity acts into the page. (a) is the non-magnetic case $(L e=0)$; (b) and (c) have a horizontal mean field $(L e=0.1)$. (a) and (b) show contours of $u_{z}$ in the plane $x=0$ with field lines overlaid in black; (c) shows a 3D isosurface of $\left|u_{z}\right|$ at $8 \%$ of its maximum value. Red (blue) indicates $u_{z}>0\left(u_{z}<0\right)$.

buoyant blob in the absence of a magnetic field, dominated by a pair of columnar vortices above the blob - one cyclonic, one anticyclonic - matched by a cyclone-anticyclone pair beneath it. However, there is also clear evidence of off-axis radiation.

Figure 2 shows the energy density $e=\frac{1}{2}\left(\boldsymbol{u}^{2}+\boldsymbol{b}^{2}\right)$ in the $y$ - $z$ plane at various times, restricted to the quadrant $y>0, z>0$. The intensity of the colour is a measure of $e$, whilst the hue is determined by $\lambda=\left(\boldsymbol{u}^{2}-\boldsymbol{b}^{2}\right) /\left(\boldsymbol{u}^{2}+\boldsymbol{b}^{2}\right)$, as indicated in the rightmost panel. Pure inertial waves are characterised by $\lambda=1$ (yellow), hybrid inertialAlfvén waves by $\lambda=0$ (green-blue) and magnetostrophic waves by $\lambda \rightarrow-1$ (dark blue). As expected, the inertial waves emerge first, inertial-Alfvén waves second, and magnetostrophic waves last, though the colour pattern is complicated by the fact that slower (more off-axis) inertial waves propagate at a similar speed to the inertial-Alfvén waves, and so the two overlap in space.

We remark that this structure bears some similarity to that found by Jault (2008), who studied the axisymmetric response of the earth's outer core to an impulsive rotation of the inner core. In both cases, the waves elongate axially before migrating radially along magnetic field lines. However, it is not clear whether quasi-geostrophy is established by inertial-Alfvén waves (rather than pure inertial waves) in Jault (2008), but they may be responsible. 
Inertial-Alfvén Waves in Planetary Cores

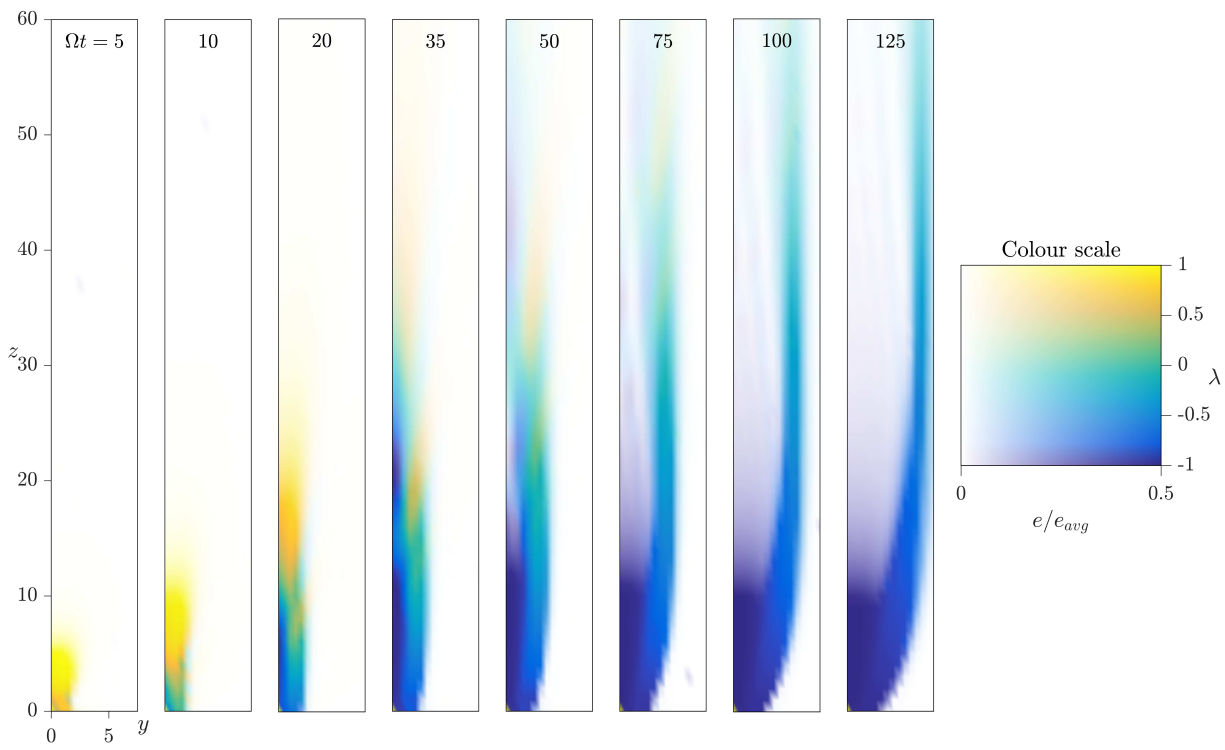

Figure 2. The energy density $e$ in the $y$ - $z$ plane at various times, for $L e=0.1$, coloured by $\lambda=\left(\boldsymbol{u}^{2}-\boldsymbol{b}^{2}\right) /\left(\boldsymbol{u}^{2}+\boldsymbol{b}^{2}\right)$ in order to distinguish the three classes of wave.
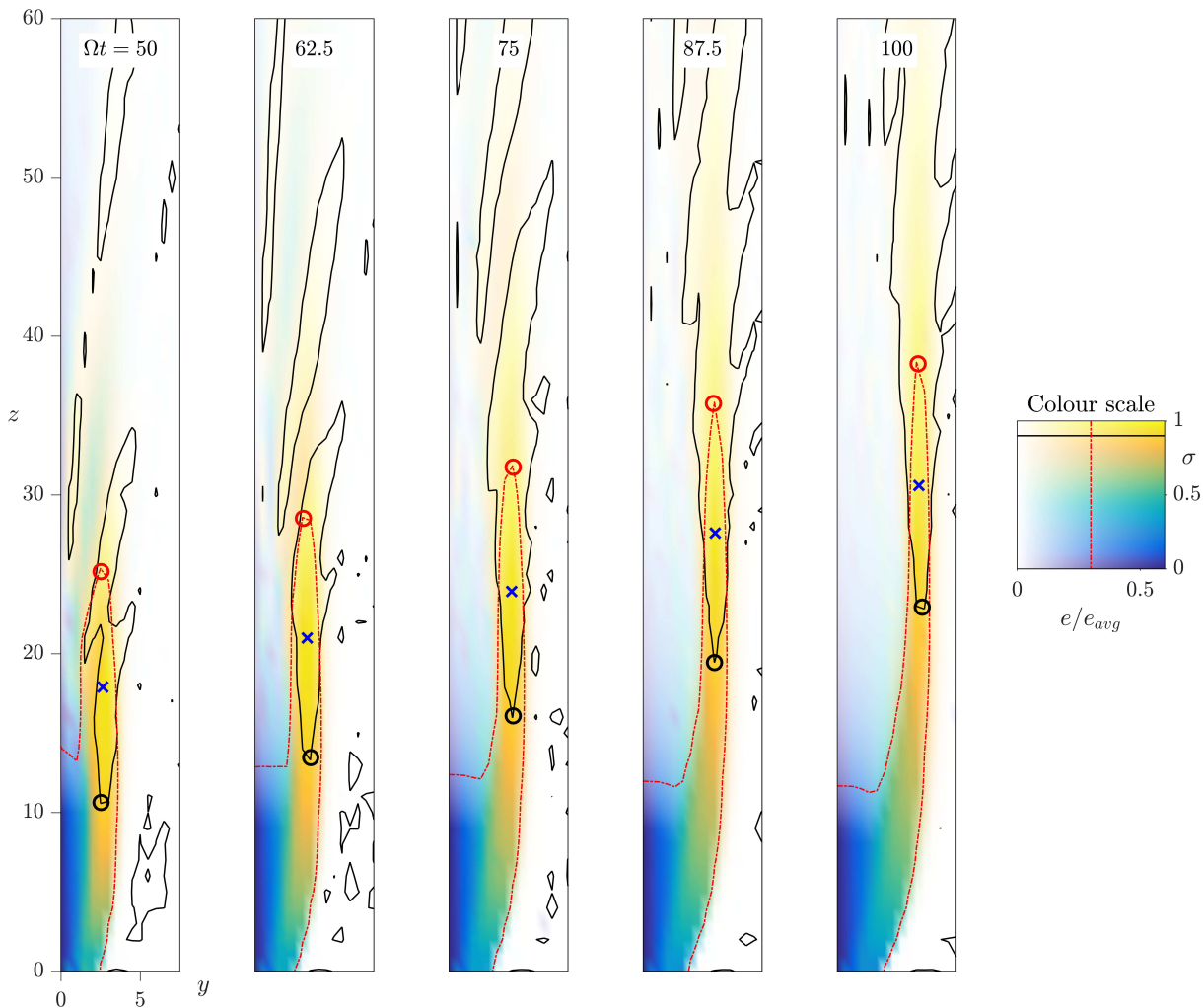

Figure 3 . The energy density $e$ at various times, coloured by normalised cross helicity $\sigma$. The solid black line marks a contour at $\sigma=0.9$, with its lowermost tip highlighted by a black circle, and the dashed red line marks a contour at $e=0.3 e_{\text {avg }}$, with its uppermost tip highlighted by a red circle. The blue cross marks the 'centre' of the wave-packet. 

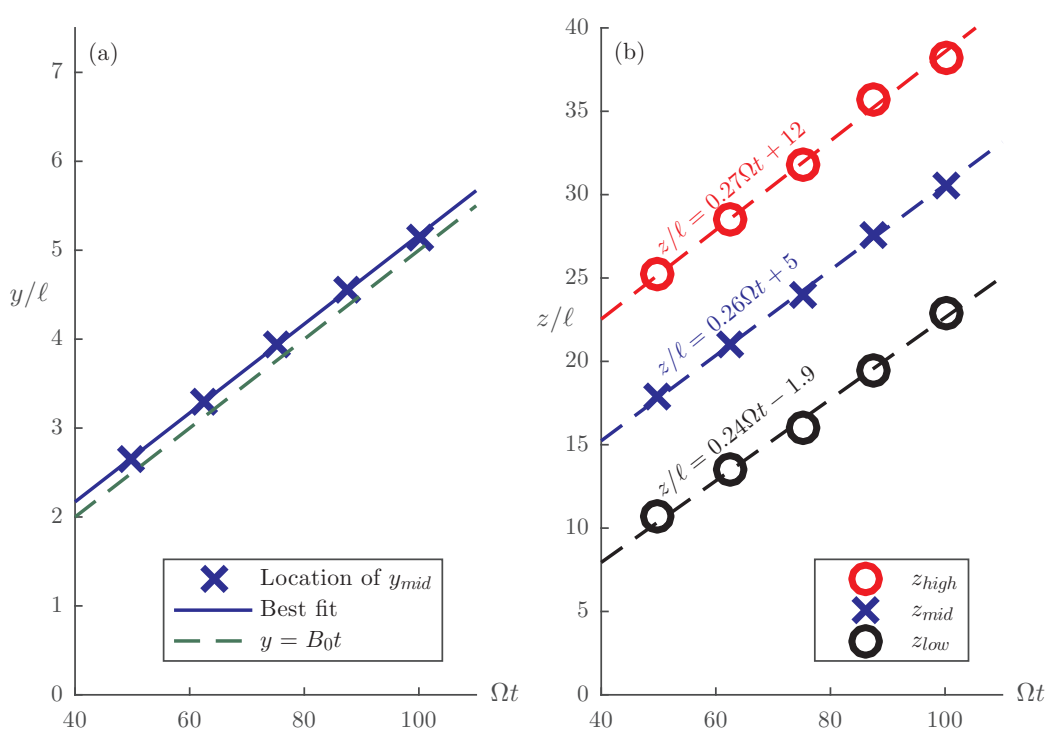

Figure 4. Spatio-temporal evolution of the inertial-Alfvén wave packet identified in figure 3. (a) shows the centre moving at the Alfvén velocity along field lines, and (b) tracks its axial velocity; markers correspond to those in figure 3 .

Figure 3 also shows the energy density, only this time coloured by the normalised cross helicity $\sigma=|\boldsymbol{u} \cdot \boldsymbol{b}| / e$. Both inertial and magnetostrophic waves are characterised by $\sigma \approx 0$ (blue/purple) and hybrid inertial-Alfvén waves by $\sigma=1$ (yellow). The solid black line indicates $\sigma=0.9$ and the dashed red line $e=0.3 e_{\text {avg }}$ (where $e_{\text {avg }}$ is an average over all space and time for the five plots shown); we take the area bounded above by $e=0.3 e_{\text {avg }}$ and below by $\sigma=0.9$ as the region of space occupied by energetic inertial-Alfvén waves, with its centre halfway between the two extrema. This provides an objective means of tracking (some fraction of) the inertial-Alfvén wave-packet as a function of time.

From figure 3 we can track the $y$ - $z$ location of the centre of our inertial-Alfvén wave packet, and this is shown as a function of $\Omega t$ in figure 4 . The left-hand panel shows that the centre of the wave-packet propagates along magnetic field lines at the Alfvén speed $\boldsymbol{B}_{0}$, consistent with $(2.1 \mathrm{~b})$, whilst the right-hand panel shows it propagating with a mean axial velocity of $c_{g z} \simeq 0.26 \Omega \ell$. Adopting the approximate result that the dominant wavenumber for a Gaussian disturbance is $k \approx \pi / \ell$, this translates to $c_{g z} \simeq 0.81 \Omega / k$, close to the prediction of (2.1b).

Finally, figure 5 shows the three-dimensional distribution of axial velocity $u_{z}$ at $\Omega t=50$ associated with a set of $10^{4}$ Gaussian blobs with centres randomly distributed over the $x-y$ plane and in the range $-2 \ell<z<2 \ell$. The dispersion pattern is dominated by cycloneanticyclone pairs, and looks very much like the images obtained from full numerical simulations of planetary dynamos. Using $\ell \sim 10 \mathrm{~km}$ (Davidson 2014), the box height would be $400 \mathrm{~km}$, so this is only valid in a local sense - as the waves propagate to larger $z$ we expect both dissipation and reflection to play a role. 


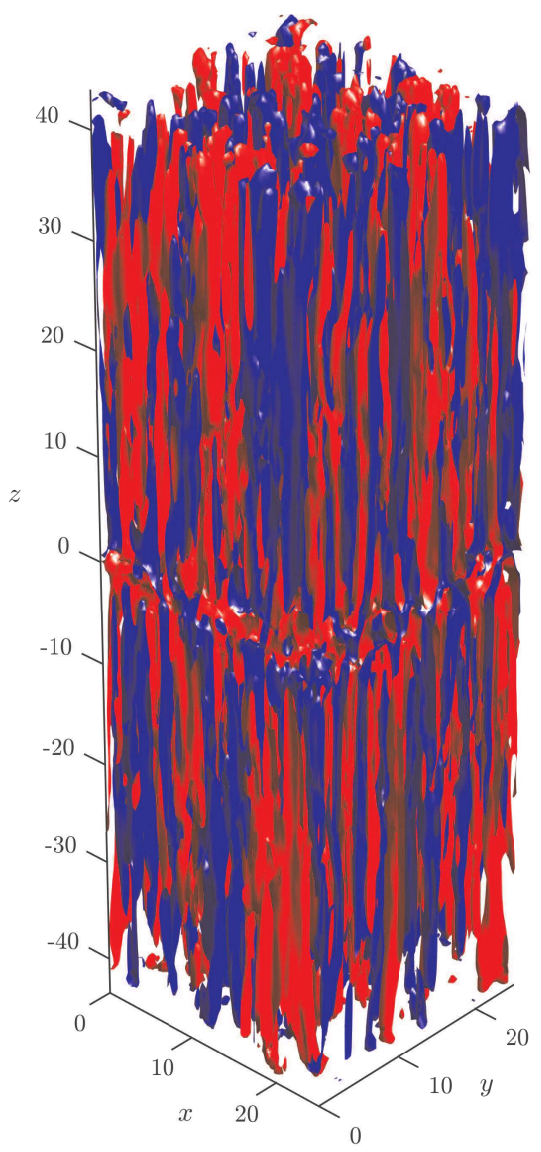

Figure 5. Axial velocity distribution at $\Omega t=50$, visualised by an isosurface of $\left|u_{z}\right|$ at $10 \%$ of its maximum value coloured red (blue) for $u_{z}>0\left(u_{z}<0\right)$, for $10^{4}$ Gaussian buoyant blobs randomly distributed in the vicinity of the $x-y$ plane. Rotation is vertical, ambient magnetic field in the $y$-direction, and gravity acts in the negative $x$-direction. Axes in units of $\ell$.

\section{Discussion}

The numerical simulations of planetary dynamos suggest a flow structure which is quasi-geostrophic and dominated by cyclonic-anticyclonic columnar vortex pairs (Olson et al. 1999). Moreover, this flow pattern is constantly changing in response to the evolving density field. Clearly this temporal evolution requires some dynamic mechanism which continually re-establishes approximate geostrophy on a time scale which is fast by comparison with the convective time scale. It is commonly assumed that the maintenance of approximate geostrophy is enforced by fast inertial waves; by way of contrast, we have shown here that, in the core of a planet, it is more likely that the columnar vortices are established by hybrid inertial-Alfvén waves which also operate on a fast time scale.

This work was undertaken with funding from an EPSRC doctoral studentship. The authors thank Avishek Ranjan and three anonymous reviewers for many helpful comments. 


\section{REFERENCES}

Braginsky, S. I. 1967 Magnetic Waves in the Earth's Core. Geomagnetism and Aeronomy 7, 851.

Christensen, U. R. 2011 Geodynamo models: Tools for understanding properties of Earth's magnetic field. Physics of the Earth and Planetary Interiors 187 (3-4), 157-169.

Chulliat, A., Loper, D. E. \& Shimizu, H. 2004 Buoyancy-driven perturbations in a rapidly rotating, electrically conducting fluid: part III - effect of the lorentz force. Geophysical \& Astrophysical Fluid Dynamics 98 (6), 507-535.

Davidson, P. A. 2013 Turbulence in Rotating, Stratified and Electrically Conducting Fluids. Cambridge University Press.

Davidson, P. A. 2014 The Dynamics and Scaling Laws of Planetary Dynamos Driven by Inertial Waves. Geophysical Journal International 198 (3), 1832-1847.

Davidson, P. A. \& RANJAn, A. 2015 Planetary dynamos driven by helical waves - II. Geophysical Journal International 202 (3), 1646-1662.

Davidson, P. A., Staplehurst, P. J. \& Dalziel, S. B. 2006 On the evolution of eddies in a rapidly rotating system. Journal of Fluid Mechanics 557, 135-144.

Finlay, C. C., Dumberry, M., Chulliat, A. \& Pais, M. A. 2010 Short Timescale Core Dynamics: Theory and Observations. Space Science Reviews 155 (1-4), 177-218.

Hide, R. 1966 Free Hydromagnetic Oscillations of the Earth's Core and the Theory of the Geomagnetic Secular Variation. Philosophical Transactions of the Royal Society of London A: Mathematical, Physical and Engineering Sciences 259 (1107), 615-647.

JAULT, D. 2008 Axial invariance of rapidly varying diffusionless motions in the Earth's core interior. Physics of the Earth and Planetary Interiors 166 (1-2), 67-76.

Moffatt, H. K. 1978 Magnetic field generation in electrically conducting fluids. Cambridge: Cambridge University Press.

Moffatt, H. K. \& Loper, D. E. 1994 The Magnetostrophic Rise of A Buoyant Parcel In the Earth's Core. Geophysical Journal International 117 (2), 394-402.

Olson, P., Christensen, U. \& Glatzmaier, G. A. 1999 Numerical modeling of the geodynamo: Mechanisms of field generation and equilibration. Journal of Geophysical Research: Solid Earth 104 (B5), 10383-10404.

St Pierre, M. G. 1996 On the local nature of turbulence in Earth's outer core. Geophysical \&6 Astrophysical Fluid Dynamics 83 (3-4), 293-306. 